\title{
Interprofessional collaboration: A gastrointestinal unit case study
}

\author{
Yiying Fang*1, Yulin Feng ${ }^{2}$, Yan $\mathrm{Xu}^{1}$ \\ ${ }^{1}$ Shanghai Renji Hospital, Shanghai, China \\ ${ }^{2}$ Shanghai Ruijin Hospital, Shanghai, China
}

Received: December 19, 2017

Accepted: April 13, 2018

Online Published: April 24, 2018

DOI: $10.5430 /$ jnep.v8n9p73

URL: https://doi.org/10.5430/jnep.v8n9p73

\begin{abstract}
Interprofessional collaboration plays a vital role in improving patient outcomes. The ability to work with professionals from different disciplines is considered a critical element of interprofessional practice. In this article, a case of a patient with upper gastrointestinal tract hemorrhage in a gastrointestinal unit is reported and analyzed from the perspective of interprofessional practice. Strategies are also introduced in the article. Enhancing mutual understanding and communication skills is conductive to interprofessional collaboration. Meanwhile, interprofessional education, as another strategy, is proved to improve the quality of health care by enhancing the cooperation among different medical team members.
\end{abstract}

Key Words: Case report, Interprofessional collaboration, Interprofessional education, Gastrointestinal Unit

\section{INTRODUCTION}

Among different healthcare providers, effective communication and teamwork are required to ensure the quality of patient care and safety. Interprofessional care occurs "when multiple health workers from different professional backgrounds provide comprehensive services by working with patients, their families, caregivers and communities to deliver the highest quality of care across settings" ${ }^{[1]}$ In recent years, interprofessional collaboration, which is regarded as the key to safe, high-quality, and accessible patient-centered care, has been developed in many countries. The ability of working with professionals from other disciplines to deliver patient-centered care is considered a critical element of interprofessional practice. Specific competencies and strategies are indispensable when concerning improving the quality of interprofessional care. However, in China, studies about interprofessional collaboration are reported rarely in the liter- ature. The purpose of this article is to present and analyze a case which occurred in a gastrointestinal (GI) unit, followed by several strategies to improve interprofessional collaboration.

\section{Case PResentation}

The case was about a clinical error, which resulted from a variety of problems among health care providers from different disciplines. $\mathrm{Mr} \mathrm{X}$, a 56-year-old male patient with a chief complaint of black tarry stools of two days duration, was admitted to GI unit of hospital Y, a tertiary hospital in Shanghai, on April 10th, 2017. He was diagnosed as upper gastrointestinal tract hemorrhage, using continuous 24-hour ECG monitoring. On April 15th, Mr X told Doctor D, who had been working in GI unit for two years, that he felt nausea. Doctor D prescribed esomeprazole, a proton pump inhibitor (PPI), for Mr X. He wanted to ask Nurse N, a nurse with

*Correspondence: Yiying Fang; Email: fangyiying88@ 126.com; Address: Shanghai Renji Hospital, Shanghai, China. 
five-year working experience, to inject for the patient, but he could not find her, because she was busy taking take of another patient. Then, Doctor D let Ms S, a nursing student, find and tell Nurse $\mathrm{N}$ to give the injection for the patient. However, after 20 minutes, Nurse $\mathrm{N}$ still did not inject for $\mathrm{Mr} \mathrm{X}$. In fact, the nursing student failed to inform Nurse $\mathrm{N}$ of the order, since she assumed that Nurse $\mathrm{N}$ would notice it soon. Therefore, the patient kept complaining to Doctor $\mathrm{D}$, and Doctor D went to the drug cupboard, found the "esomeprazole", and injected for Mr X by himself. He was so exasperated by Nurse $\mathrm{N}$ that he did not check the ampoule with her before injection, even though she asked if she needed to check. At last, without checking the drug, Doctor D injected Mr X with omeprazole, another PPI, instead of esomeprazole. The doctor realized the wrong injection he gave, but insisted that it was partially the nurse's fault, as she did not inject for the patient as required. Doctor D was going to admit the error to $\mathrm{Mr} \mathrm{X}$, but intended to communicate with Nurse $\mathrm{N}$ and the nursing student first.

\section{Case analysis}

Interprofessional teamwork, which can enhance cooperation, coordination, and collaboration and increase patient satisfaction, when healthcare providers deliver patient-centered care, is becoming the forefront of modern healthcare system. In the case above, there are a variety of issues such as misunderstanding and ineffective communication that are responsible for the medical error. For example, the nursing student failed to inform the nurse about the patient; the doctor did not deliver request by a normal communication method to alert the nurse; the doctor ignored the nurse's request of checking the drug; the doctor and the nurse misunderstood each other's roles and responsibilities. These collaboration failures ultimately resulted in the clinical error According to the Interprofessional Education Collaborative (IPEC) report, ${ }^{[2]}$ there are four competencies of interprofessional practice. They are values/ethics for interprofessional practice, roles/responsibilities, interprofessional communication, and teams and teamwork. Further issues are discussed based on these four competencies.

\subsection{Values/ethics for interprofessional practice}

Healthcare providers in an interprofessional team are from different professional areas. They have different values, and bring the values of their professions to the team. ${ }^{[3]}$ The values of a profession guide and shape a healthcare provider's decision-making and interactions with patients and coworkers. ${ }^{[4,5]}$ In the case above, the healthcare professionals had limited knowledge of each other's values, and thus led to value conflicts. Lack of contact can result in value conflicts and have the potential to affect team collaboration and func- tioning, since professions have few opportunities to explore or learn about values from each other. ${ }^{[6]}$ Core to identifying and managing these conflicts is knowledge of the values of the other professions in the interprofessional team, and acknowledging and respecting the expertise of all professionals are of great importance regarding the benefit of the patients. ${ }^{[7]}$ Therefore, healthcare professionals are required to develop a trusting relationship with other team members, respect their cultures, values and expertise, and act with honesty in the relationship.

\subsection{Roles/responsibilities}

Understanding and appreciating roles and responsibilities of different professionals are two competencies of collaborative practice. ${ }^{[8]}$ Generally speaking, it is a nurse's responsibility to check drugs, for nurses are trained to check drugs carefully, and they are often the last opportunity to avoid an error, although doctors are also supposed to check. Meanwhile, injection is a nurse's work rather than a doctor's, although the doctor also should be responsible for what they have injected for the patient.

Studies indicate that a good understanding and appreciation of roles and responsibilities lead to better patient outcomes, well-functioning teams, and higher job satisfaction. ${ }^{[9]}$ Interprofessional collaboration is an interdependent relationship, which requires a recognition of complementary roles, and a respect for each profession's scope of knowledge and abilities. Effective collaborative practice could only happen in an environment of mutual trust and respect. Clarifying each member's roles and responsibilities can help team members find their own places in the team and recognize each other's strength. Lack of communication with team members about each other's roles and responsibilities is considered an obstacle to build an interdependent relationship and provide high-quality health care services. ${ }^{[8]}$

\subsection{Interprofessional communication}

Effective communication, which involves multiple components, is described in literature as a core competency for collaborative practice. ${ }^{[8]}$ Researchers have found that there is a link between communication and patient outcomes, ${ }^{[10]}$ Communication failures lead to interruptions in the continuity of care and have the potential to harm the patients, and are therefore a threat to patient safety. ${ }^{[11]}$ Incomplete and delayed information due to ineffective communication has been proved to adversely affect the management of patient care. ${ }^{[12]}$ Meanwhile, the ability to choose effective communication tools and techniques, including communication technologies and information systems, can enhance team functions and interactions among team members, whereas the absence of 
electronic communication tools as mentioned has negative influence on effective collaborative communication, ${ }^{[13]}$ which has been found to increase adverse clinical outcomes and lower the quality of care. ${ }^{[12]}$

\subsection{Teams and teamwork}

Interprofessional care is a kind of team-based practice. Teamwork plays a vital role in the process of interprofessional practice, which helps to engage health professionals in, and integrate their knowledge and strengths. In the case, all the health professionals acted as individuals, not as a team. For example, the doctor did not want to check drugs with the nurse before injection. In fact, no single profession can provide all the information needed to deal with complex patient conditions. An effective interprofessional collaboration requires team members to work together to achieve the shared goals, even though the team members have their own tasks. Moreover, teamwork provides health care professionals with opportunities of collective learning and development through working together, sharing ideas, making decisions, and solving problems. This can help a team become more efficient and able to create more appropriate methods of working. ${ }^{[14]}$

Furthermore, leadership plays an important role in interprofessional practice. In this case, there were only two nurses and one doctor involved in the patient's care, and there was no team leader, who should be a key person in the team. Studies show that a lack of clear leadership is negatively associated with team development. ${ }^{[15]}$ A leader in an interprofessional team will influence the team's vision, direction and objectives, encourage collective learning, coordinate the team, supervise and support team members. Supportive leadership practices can enhance collaboration and team functions.

\section{IDENTIFIED STRATEGIES}

To enhance the quality of interprofessional care, strategies should be developed and applied in practice. Based on the case analysis above, the strategies include enhancing mutual understanding, communication skills training and interprofessional education.

\subsection{Enhancing mutual understanding}

Physicians, nurses, pharmacists, psychologists, dieticians and other professionals are involved in a collaborative team and play a vital role in patient care. Recognition of other professionals' values is a significant prerequisite for collaboration. Strategies for interprofessional collaboration begin with mutual understanding, which is a basis of interprofessional collaboration. It is important for health professionals to identify their values, roles and responsibilities and make them known by other team members, for value conflicts and misunderstanding of roles and responsibilities among members have the potential to affect team collaboration. With in-depth understanding of their own and others' values, roles and responsibilities, healthcare providers will be able to know more about others' backgrounds and skills, and promote an effective interprofessional collaboration. ${ }^{[16]}$ To enhance mutual understanding, multi-discipline team (MDT) meetings, which involves professions from a variety of disciplines, is recommended to be held regularly. The meetings provide healthcare providers with opportunities for interprofessional interaction, as they can discuss complicated symptoms, analyze lab test results, or check a patient's mental status together. ${ }^{[17]}$

\subsection{Improving communication skills}

Communication skills can be regarded as the core competency in interprofessional practice. Another strategy, therefore, is improving communication skills, which is perceived to positively influence the quality of patient care. ${ }^{[18]}$ To ensure patient safety, improve patient satisfaction, effective communication skills are required, when healthcare professionals are delivering patient care. Evidence also suggests that communication skill training courses are recommended in hospital settings, which can enhance healthcare professionals' supportive and communication skills. ${ }^{[19]}$ Other methods, such as active learning, peer teaching, providing opportunities to express ideas and opinions, and encouraging to use computer communication systems, are also useful in terms of developing both communication and interaction skills. ${ }^{[8]}$

\subsection{Interprofessional education}

Interprofessional education (IPE) is designed to provide health care workers from different professions with opportunities to work together, learn knowledge, attitudes and skills from each other, and thus enhance future collaboration. ${ }^{[20]}$ IPE activities, such as simulation-based education programs, patient-centered case studies, health promotion activities and interactive lectures, are proved to be strategies for developing and strengthening interprofessional competencies. ${ }^{[21]}$ In addition, educators are also recommended to develop curricula that can provide medical students of different majors with opportunities to learn from each other, since those curricula not only enhance their collaboration and teamwork skills, but also support them to know other professionals' roles and responsibilities, and thus improve patient outcomes.

\section{Conclusion}

The case, which is about a clinical error, has presented a variety of interprofessional problems among health workers from different professions, including problems of values, roles and 
responsibilities, communication and teamwork. To enhance the quality of interprofessional care, health professionals should recognize others' values, roles and responsibilities, enhance communication and encourage teamwork. Strategies, such as developing mutual understanding, improving communication skills and providing interprofessional edu- cation, can be applied into interprofessional practice, and thus improve patient outcomes. Moreover, nurse educators are encouraged to develop curricula about interprofessional practice, and provide communication skills training courses.

\section{CONFlicts OF INTEREST Disclosure}

The authors declare that there is no conflict of interest.

\section{REFERENCES}

[1] Gilbert JH, Yan J, Hoffman SJ. A WHO report: framework for action on interprofessional education and collaborative practice. J Allied Health. 2010; 39(Suppl 1): 196-197. PMid:21174039

[2] Interprofessional Education Collaborative Expert Panel. Core competencies for interprofessional collaborative practice: Report of an expert panel. Washington, D.C.: Interprofessional Education Collaborative. 2011.

[3] Hall P. Interprofessional teamwork: professional cultures as barriers. J Interprof Care. 2005; 19(Suppl 1): 188-196. PMid:16096155 https://doi.org/10.1080/13561820500081745

[4] Duque RL. Health Promotion and the Values of Occupational Therapy. World Federation of Occupational Therapists Bulletin. 2004; 49(1): 5-8. https://doi.org/10.1179/otb.2004.49.1.002

[5] Kanny E. Core Values and Attitudes of Occupational Therapy Practice. Am J Occupational Therapy. 1993; 47(12): 1085-1086. https://doi.org/10.5014/ajot.47.12.1085

[6] Erickson B, McHarney-Brown C, Seeger K, et al. Overcoming barriers to interprofessional health sciences education. Educ for Health. 1998; 11(2): 143-149.

[7] Orchard CA, Curran V, Kabene S. Creating a culture for interdisciplinary collaborative professional practice. Med Educ Online. 2005; 10(1): 4387. PMid:28253153 https://doi.org/10.3402/meo. v10i.4387

[8] Suter E, Arndt J, Arthur N, et al. Role understanding and effective communication as core competencies for collaborative practice. J Interprof Care. 2009; 23(1): 41-51. PMid:19142782 https: //doi.org/10.1080/13561820802338579

[9] Griffin MA, Patterson MG, West MA. Job satisfaction and teamwork: the role of supervisor support. J Occup Behav. 2001; 22(5): 537-550. https://doi.org/10.1002/job.101

[10] Stewart M, Brown JB, Donner A, et al. The impact of patient-centred care on outcomes. J Fam Pract. 2000; 49(9): 796-804.

[11] Root causes of Sentinel Events. Joint Commission on Accreditation of Healthcare Organizations. Available from: http://www . joint commission.org/NR/rdonlyres/FA46564 6-5F5F-4543-AC8F-E8AF6571E372/0/root_cause_se.jpg

[12] Kripalani S, Lefevre F, Phillips CO, et al. Deficits in communication and information transfer between hospital-based and primary care physicians: implications for patient safety and conti- nuity of care. JAMA. 2007; 297(8): 831-841. PMid:17327525 https://doi.org/10.1001/jama.297.8.831

[13] Chatalalsingh C, Reeves S. Leading team learning: what makes interprofessional teams learn to work well? J Interprof Care. 2014; 28(6): 513-518. PMid:24654793 https ://doi . org/10.3109/13 561820.2014 .900001

[14] Sims S, Hewitt G, Harris R. Evidence of a shared purpose, critical reflection, innovation and leadership in interprofessional healthcare teams: a realist synthesis. J Interprof Care. 2015; 29(3): 209215. PMid:25051089 https://doi.org/10.3109/13561820.2 014.941459

[15] Haward R, Amir Z, Borrill C, et al. Breast cancer teams: the impact of constitution, new cancer workload, and methods of operation on their effectiveness. Br J Cancer. 2003; 89(1): 15-22. PMid:12838294 https://doi.org/10.1038/sj.bjc.6601073

[16] Pecukonis E, Doyle O, Bliss DL. Reducing barriers to interprofessional training: promoting interprofessional cultural competence. J Interprof Care. 2008; 22(4): 417-428. PMid:18800282 https : //doi.org/10.1080/13561820802190442

[17] Bridges DR, Davidson RA, Odegard PS, et al. Interprofessional collaboration: three best practice models of interprofessional education. Med Educ Online. 2011 Jan; 16(1): 1-10.

[18] Kerber KA, Hofer TP, Meurer WJ, et al. Emergency department documentation templates: variability in template selection and association with physical examination and test ordering in dizziness presentations. BMC Health Serv Res. 2011; 11: 65. PMid:21435250 https://doi.org/10.1186/1472-6963-11-65

[19] Moore PM. Communication skills training for healthcare professionals working with people who have cancer. Rivera Mercado S, Grez Artigues M, Lawrie TA, editors. Cochrane Database Syst Rev[Internet]. 2015 Apr 1. Available from: http://ezproxy. chatham. edu : 2048/login?url=http: $/ /$ search.ebscohost. com/login. aspx?direct $=$ true\&db=ch $\mathrm{h} \& \mathrm{AN}=\mathrm{CD} 003751 \&$ site $=$ eds - live\& $\mathrm{cope}=$ site

[20] Hall P, Weaver L. Interdisciplinary education and teamwork: a long and winding road. Med Educ. 2001; 35(9): 867-875. https : //doi.org/10.1046/j.1365-2923.2001.00919.x

[21] Khan NS, Shahnaz SI, Gomathi, KG. Currently Available Tools and Teaching Strategies for the Interprofessional Education of Students in Health Professions: Literature review. Sultan Qaboos Univ Med J. 2016; 16(4): e277-285. PMid:27606105 